\title{
Subclinical atherosclerosis among rheumatoid arthritis patients without overt cardiovascular risk factors
}

\begin{abstract}
Objective. To determine the associated factors of subclinical atherosclerosis measured with carotid intima media thickness (CIMT) among rheumatoid arthritis (RA) patients without any overt traditional cardiovascular $(\mathrm{CV})$ risk factors. Methods. Forty RA patients with matched age and gender healthy controls were recruited. Carotid ultrasound was performed to all subjects. CIMT was considered to be abnormally thickened if it was more than the 75th percentile matched for age and sex reference values. Univariate and multivariate analyses were performed to determine the association between the sociodemographics and disease characteristics of RA with thickened CIMT. Results. Abnormally thickened CIMT were observed in 11 RA patients $(27.5 \%)$ and in 4 control subjects $(10 \%), p=0.04$. It was highly prevalent among RA patients with active disease $(54.5 \%$ vs $17.2 \%), p=0.02$. Patients with thickened CIMT also tend to have erosive disease, $\mathrm{p}=0.06$. Seropositive rheumatoid factor (RF) patients also had significantly higher CIMT values as compared with sero-negative patients, $\mathrm{p}=0.03$. Multivariable logistic regression analysis revealed that active disease was independently associated with thickened CIMT. Conclusions. RA patients are at risk for subclinical atherosclerosis despite absence of traditional CV risk co morbidities and active disease was the independent factor associated with it.
\end{abstract}

Keyword: Atherosclerosis; Carotid intima media thickness; Disease activity; Rheumatoid arthritis 